\title{
Genetic and Pathogenic Characterization of Bacterial Wilt Pathogen, Ralstonia pseudosolanacearum (Ralstonia solanacearum Phylotype I), on Roses in Korea
}

\author{
Ingyeong Lee ${ }^{1}$, Yeong Son Kim ${ }^{1}$, Jin-Won Kim ${ }^{2}$, and Duck Hwan Park (iD ${ }^{1 *}$ \\ ${ }^{1}$ Applied Biology Program, Division of Bioresource Science, College of Agriculture and Life Sciences, Kangwon \\ National University, Chuncheon 24341, Korea \\ ${ }^{2}$ Department of Environmental Horticulture, University of Seoul, Seoul 02504, Korea
}

(Received on June 5, 2020; Revised on August 27, 2020; Accepted on September 7, 2020)

The purpose of this study was to analyze the genetic and pathogenic characteristics of Ralstonia pseudosolanacearum in roses in Korea, and to examine the similarities and differences between Korean isolates and the first-reported European strains. Between 2017 and 2019, seventeen isolates from rose plants were identified as $\boldsymbol{R}$. pseudosolanacearum using Ralstonia-specific primers. All 17 isolates were identified as race 1 using race-specific primers, and were confirmed as biovar 3 due to their ability to utilize carbon sources. Multiplex PCR using phylotype discriminating specific primers identified the 17 isolates as phylotype I. Sequevar comparison with reference sequevars using the sequences of the $e g l$, mutS, and $f l i C$ genes, and only the $e g l$ gene, revealed that the strains evaluated in this study corresponded to sequevar I-33. The pathogenicity in roses differed depending on the rose cultivars. The different methods used for the genetic characterization of $\boldsymbol{R}$. pseudosolanacearum indicate that the 17 rose bacterial wilt isolates had the same genetic characteristics. The lack of genetic variation in these isolates indicates their recent introduction from other countries (likely European countries). Therefore, appropriate quarantine and control measures should be taken in order to avoid

*Corresponding author.

Phone) +82-33-250-6432, FAX) +82-33-259-5558

E-mail)dhp@kangwon.ac.kr

ORCID

Duck Hwan Park

https://orcid.org/0000-0001-8486-9544

(c) This is an Open Access article distributed under the terms of the Creative Commons Attribution Non-Commercial License (http:// creativecommons.org/licenses/by-nc/4.0) which permits unrestricted noncommercial use, distribution, and reproduction in any medium, provided the original work is properly cited.

Articles can be freely viewed online at www.ppjonline.org. further increases in the pathogenicity and/or secondary host range of $\boldsymbol{R}$. pseudosolanacearum through genetic mutation.

Keywords : pathogenicity, phylotype, rose, Ralstonia pseudosolanacearum, sequevar

Handling Editor : Nai-Chun Lin

In 2015, an unknown disease with atypical symptoms was reported in roses (Rosa sp. cv. Armando, Sativa, and Red Naomi) in the Netherlands. These symptoms differed from those caused by fungal pathogens reported previously on roses, and included wilting of young shoots and flower stalks, yellowing of leaves, and dieback of pruned branches. The causal pathogen from diseased rose samples was confirmed to be Ralstonia solanacearum, and this record of bacterial wilt in roses caused by $R$. solanacearum was the first of its kind anywhere in the world (Tjou-TamSin et al., 2017a). As soon as bacterial wilt disease was confirmed in roses, researchers and members of the Ministry of Agriculture in the Netherlands realized this posed a serious problem for growers and distributors of roses, and worked to quickly characterize the pathogen and identify its positioning of classification. For example, Tjou-TamSin et al. (2017b) found that the pathogenic abilities of the causal agent, $R$. solanacearum phylotype I ( $R$. pseudosolanacearum) in a variety of rose cultivars depended on inoculation methods and temperatures. They identified two susceptible cultivars, and the sensitivity of both cultivars to infection with $R$. pseudosolanacearum increased when they were exposed to the pathogen by stem inoculation after wounding, and at a temperature of $28^{\circ} \mathrm{C}$. These results indicate that this pathogenic bacterium has optimum conditions under which symptoms appear on roses, and management 
techniques involving usage of resistant cultivars and methods to avoid scars produced during leaf detaching should be applied. In addition, hierarchical classifications of 129 $R$. pseudosolanacearum strains isolated from roses in the Netherlands were conducted based on the phylotype and sequevar, and results were compared with an isolate from India (Bergsma-Vlami et al., 2018). Bergsma-Vlami et al. (2018) concluded that all strains were grouped into phylotype I and sequevar 33, which were well-matched with the strain from India, and therefore all strains were found to belong to the same monophyletic group. Studies aimed at evaluating the genetic classification and pathogenicity of $R$. pseudosolanacearum have aimed to develop to cope with this emerging pathogen in the Netherlands.

After R. pseudosolanacearum was first reported from roses in the Netherlands, bacterial wilt disease was recorded in two rose production sites in Switzerland in 2016. Rose seedling imported by three companies in Switzerland were also suspected to be infected with $R$. solanacearum (EPPO Reporting Service no. 04-2017). However, eradication measures were implemented as soon as the pathogen was confirmed to be $R$. solanacearum race 1 , and Switzerland's National Plant Protection Organization (NPPO) declared that it had successfully eradicated bacterial wilt in roses in Switzerland in 2018 (EPPO Reporting Service no. 10-2018).

In 2017, wilting and yellowing symptoms were observed on leaves of rose plants grown on large flower farms in Korea. The causal pathogen was reported to be $R$. solanacearum phylotype I ( $R$. pseudosolanacearum) making Korea the third country in the world following the Netherlands and Switzerland to record $R$. pseudosolanacearum infections in roses (Kim et al., 2019). It is likely that $R$. pseudosolanacearum infections in roses spread to Korea through the importation of propagating plant materials such as root stocks and branches for cut flowers from European countries such as the Netherlands. To confirm this, the causal pathogens of bacterial wilt on roses from Korea and the Netherlands must be compared based on their genetic characterization. Thus, this study aimed to evaluate whether the $R$. pseudosolanacearum infecting roses in Korea and the Netherlands are genetically similar. We evaluated this using traditional and hierarchical classifications such as race and biovar, and phylotype and sequevar, respectively. We also studied the pathogenic properties of $R$. pseudosolanacearum isolated from roses and a strain isolated from potatoes (Solanum tuberosum) in roses and tomatoes $(S$. lycopersicum), which are the natural host and the most sensitive to infection. As such, the two aims of our study were to characterize the phenotypic and genetic variation of $R$. pseudosolanacearum infecting Korean roses, and to evaluate the pathogenicity of $R$. pseudosolanacearum depending on host range.

\section{Materials and Methods}

Biosafety approval. Kangwon National University granted biosafety approval for the experiments conducted as part of this study, and all $R$. solanacearum and $R$. pseudosolanacearum strains were maintained under the direction of the Korean Biotechnology Industry Organization (KoreaBio).

Isolation of $\boldsymbol{R}$. pseudosolanacearum from wilting symptomatic roses. Stem cuttings of roses with wilting and dieback symptoms were collected from rose farms in Taean, Goyang, Paju, and Jangsu cities/districts. The stems of diseased roses were sliced in sections of $2 \mathrm{~cm}$, with each section including symptomatic and asymptomatic parts. Stem sections were sterilized with $70 \% \mathrm{EtOH}$ and then washed three times with distilled water. Stem sections were placed in extraction bags (Bioreba, Reinach, Switzerland) and 2 $\mathrm{ml}$ of $10 \mathrm{mM} \mathrm{MgCl}_{2}$ was added to the samples for maceration. Each $1 \mathrm{ml}$ aliquot of rose stem extract was serially diluted, and $100 \mu \mathrm{l}$ of extract was plated on modified SMSA (semi selective South Africa agar) medium and incubated at $28^{\circ} \mathrm{C}$ for 2 days (Elphinstone et al., 1996). For confirmation of $R$. pseudosolanacearum, typical pink mucoid colonies with red center were amplified with $R$. solanacearumspecific primers 759/760 (Supplementary Table 1) (Opina et al., 1997). Between 2017 and 2019, we isolated a total of 17 R. pseudosolanacearum strains from roses. Our analysis also included one $R$. solanacearum strain isolated from potatoes (Korean Agricultural Culture Collection; KACC 10697), in order to compare the phenotypes and genotypes of this strain and those isolated from Korean roses (Supplementary Table 2).

Phenotypic characterization of $R$. pseudosolanacearum. To identify the race of the $R$. pseudosolanacearum isolated from Korean roses, race-specific primers from previous studies were used (Supplementary Table 1). We included the following race-specific primers: PS-IS-F/PS-IS-R for race 1, ISRso19-F/ISRso19-R for race 2, 630/631 for race 3 , and two kinds of primers (AKIF/AKIR and 21F/21R) for race 4 (Fegan et al., 1998; Horita et al., 2004; Lee and Khor, 2003; Lee et al. 2001). However, there is no available specific primer for race 5 , and we did not test for race 5 in this study. The PCR reaction consisted of $100 \mathrm{ng} / \mu \mathrm{l}$ DNA of each strain, $400 \mathrm{mM}$ of each primer, and $12.5 \mu \mathrm{l}$ 
of 2× EmeraldAmp GT PCR Master Mix (Takara, Tokyo, Japan) in a total volume of $25 \mu$ l. Reactions were carried as described for each race-specific primer's protocol. PCR products were confirmed on $1 \%$ of agarose gel and observed under UV light.

For the biovar determination, all 17 strains isolated from Korean roses and KACC 10697 were cultured in mineral medium with $1 \%$ carbon sources (disaccharides: lactose, maltose, and cellobiose; sugar alcohols: mannitol, sorbitol, and dulcitol) at $28^{\circ} \mathrm{C}$ for two weeks, and the ability of the strains to utilize the carbon sources was evaluated (Hayward, 1964; Hayward et al., 1990). Distilled water was used as a negative control.

Genetic characterization of $\boldsymbol{R}$. pseudosolanacearum. To determine the phylotype of the $R$. pseudosolanacearum isolated included in our study, we used multiplex PCR with four kinds of forward primers for each phylotype (phylotype I, Nmult21:1F; II, Nmult21:2F; phylotype III, Nmult23:AF; and phylotype IV, Nmult22:InF) and one reverse primer (Nmult22:RR), as reported previously (Supplementary Table 1) (Fegan and Prior, 2005), as well as the $R$. solanacearum-specific primers 759/760 (Opina et

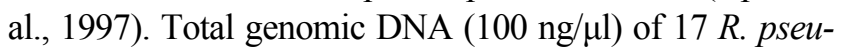
dosolanacearum isolates obtained from roses in Korea and KACC 10697 was added to $12.5 \mu \mathrm{l}$ of $2 \times$ EmeraldAmp GT PCR Master Mix, with a final volume of $25 \mu$. The PCR conditions were as follows: one pre-denaturing step at $96^{\circ} \mathrm{C}$ for $5 \mathrm{~min}$, followed by 30 cycles with denaturation at $95^{\circ} \mathrm{C}$ for $15 \mathrm{~s}$, annealing at $50^{\circ} \mathrm{C}$ for $30 \mathrm{~s}$, and extension at $72^{\circ} \mathrm{C}$ for $30 \mathrm{~s}$, and then a final extension step at $72^{\circ} \mathrm{C}$ for 10 min. PCR products were confirmed on $1 \%$ of agarose gel. Phylotypes were also determined based on a phylogenetic tree created using Mega X software (Kumar et al., 2018; Tompson et al., 2003) using 16s rRNA gene partial sequences with representative strains from each phylotype.

To evaluate the sequevars of the $17 R$. pseudosolanacearum strains isolated from Korean roses, multi locus phylogeny was applied, using three individual genes: the endoglucanase gene $e g l$, the DNA repair gene $m u t S$, and the flagellin gene $f l i C$. The analysis was also conducted using only the $e g l$ gene, as described by Bergsma-Vlami et al. (2018). The amplicons for $e g l, m u t S$, and $f l i C$ were produced with Endo-F/Endo-R, mutS-RsF1570/mutSRsR1926, and Rsol_fli_for/Rsol_fli_rev primers, respectively (Supplementary Table 2) (Poussier et al., 2000; Schönfeld et al., 2003; Wicker et al., 2007), and each sequence was trimmed using Vector NTI suit 8.0 (Thermo Scientific, Waltham, MA, USA), and aligned with ClustalW. The phylogenic tree was generated using the neigh- bor-joining method with 1,000 bootstraps in Mega X with different sequevars (Supplementary Table 3).

Pathogenicity test. The pathogenicity test was conducted in roses (cv. Fuego and cv. Hera) and tomatoes (cv. Moneymaker) grown for 4 weeks. Roses and tomatoes were inoculated with RW 078, which was chosen as a representative strain amongst the strains isolated from Korean roses. KACC 10697 and distilled water were used as a positive and negative control, respectively. Inoculations were conducted by drenching $50 \mathrm{ml}$ of bacterial suspension in $10 \mathrm{mM} \mathrm{MgCl}{ }_{2}$ buffer (approximately $10^{8} \mathrm{cfu} / \mathrm{ml}$ ) from overnight cultured on casamino acid peptone glucose (CPG) medium (Tjou-Tam-Sin et al., 2017b). Inoculated roses and tomatoes were incubated at $25^{\circ} \mathrm{C}$ and $70 \%$ relative humidity for 30 days and 14 days, respectively, to produce typical symptoms of wilt. Disease severity was recorded to the following scale: $0=$ no symptoms, $1=$ wilting on lower leaves, 2 = discoloration and up to $50 \%$ of wilting symptoms on leaves, $3=$ discoloration and upper $50 \%$ of wilting symptoms on leaves, $4=$ died for roses; $0=$ no symptoms, $1=$ up to $50 \%$ of wilting symptoms on leaves, $2=$ more than $50 \%$ of wilting symptoms on leaves, $3=$ died for tomatoes.

Statistical analysis. Two independent experiments were conducted. Disease severity was carried out by analysis of variance (ANOVA) with strain means separated by Duncan's multiple range test at $P<0.05$ using SAS software (SAS Institute, Cary, NC, USA).

\section{Results}

Isolation of $R$. pseudosolanacearum from infected roses. From May to November in 2017-2019, which are rose cultivating season in greenhouse, bacteria were isolated from roses in which stems showed signs of wilting and the lower parts of leaves showed signs of yellowing. The isolated bacteria were cultured on modified SMSA medium. Typical mucoid colonies, i.e., those that were pinkish with red centers were collected on CPG medium, and 17 isolates were confirmed as $R$. solanacearum by PCR with specific primer sets 759/760. Furthermore, all 17 strains were clustered with $R$. pseudosolanacearum strains UQRS649 and 650 based on partial 16S rRNA gene and 16S-23S rRNA internal transcribed spacer region sequences (data not shown). As such, all 17 strains isolated from roses in Korea were identified as $R$. pseudosolanacearum. Of the 17 strains, nine were isolated from greenhouses on farms in Taean, four from Goyang, two from Jangsu, and one 

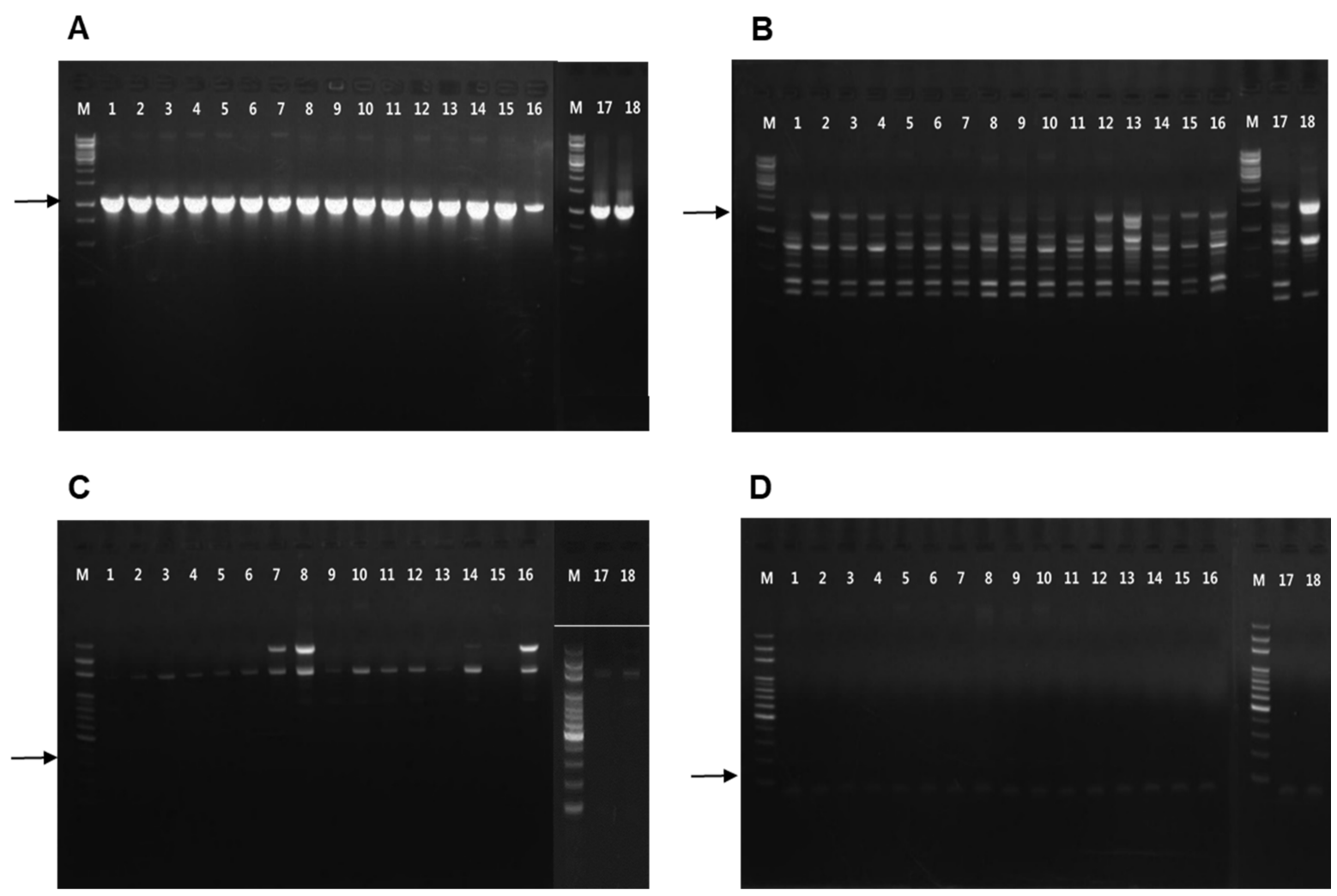

Fig. 1. Gel electrophoresis of PCR products for race determination of Ralstonia pseudosolanacearum strains isolated from Korean roses using specific primer sets for each race. (A) PCR products produced with primers PS-IS-F/PS-IS-R. The arrow indicates $1,070 \mathrm{bp}$ bands, which are consistent with race 1. (B-D) PCR products produced with specific primer sets for race 2, 3, and 4, respectively. No amplicons of expected sizes (arrows) for race 2 (1,884 bp), 3 (357 bp), and 4 (165 bp) were produced. M, $1 \mathrm{~kb}$ DNA ladder for A and B (Thermo Scientific, Waltham, MA, USA) and 100 bp DNA ladder for C and D (MGmed, Seoul, Korea); lanes 1-17, R. pseudosolanacearum isolates from Korean roses; lane 18, KACC 10697.

from Paju. The rose cultivars from which the $R$. pseudosolanacearum were isolated were cv. Fuego, cv. Hera, cv. Giorgio, and cv. Santorini for nine, four, two, and two strains, respectively.

Race and biovar determination of $R$. pseudosolanacearum strains isolated from Korea. All 17 R. pseudo- solanacearum strains isolated from Korean roses produced 1,070 bp amplicons, which was the expected amplicon size produced by the primers specific to race 1 (PS-IS-F/PS-IS$\mathrm{R})$. No products were amplified in the PCR reactions using specific primers for races 2,3 , and 4 . These results indicate that all 17 strains belonged to race 1 (Fig. 1). For biovar determination, all 17 strains were found to utilize three

Table 1. Biochemical tests for biovar determination of Ralstonia pseudosolanacearum strains isolated from Korean roses

\begin{tabular}{|c|c|c|c|c|c|c|c|c|c|c|c|c|c|c|c|c|c|c|c|c|c|c|c|c|c|}
\hline $\begin{array}{l}\text { Carbon } \\
\text { source }\end{array}$ & $\begin{array}{l}\text { RW } \\
007\end{array}$ & $\begin{array}{l}\text { RW } \\
008\end{array}$ & $\begin{array}{l}\text { RW } \\
010\end{array}$ & $\begin{array}{l}\text { RW } \\
014\end{array}$ & $\begin{array}{l}\text { RW } \\
016\end{array}$ & $\begin{array}{l}\text { RW } \\
017\end{array}$ & $\begin{array}{l}\text { RW } \\
020\end{array}$ & $\begin{array}{l}\text { RW } \\
021\end{array}$ & $\begin{array}{l}\text { RW } \\
030\end{array}$ & $\begin{array}{l}\text { RW } \\
059\end{array}$ & $\begin{array}{l}\text { RW } \\
060\end{array}$ & $\begin{array}{l}\text { RW } \\
065\end{array}$ & $\begin{array}{l}\text { RW } \\
068\end{array}$ & $\begin{array}{l}\text { RW } \\
075\end{array}$ & $\begin{array}{l}\text { RW } \\
076\end{array}$ & $\begin{array}{l}\text { RW } \\
077\end{array}$ & $\begin{array}{l}\text { RW } \\
078\end{array}$ & $\begin{array}{c}\text { KACC } \\
10697\end{array}$ & DW & & & 2 & 3 & 4 & 5 \\
\hline Maltose & + & + & + & + & + & + & + & + & + & + & + & + & + & + & + & + & + & - & & - & - & + & + & - & + \\
\hline Lactose & + & + & + & + & + & + & + & + & + & + & + & + & + & + & + & + & + & - & - & - & - & + & + & - & + \\
\hline Cellobiose & + & + & + & + & + & + & + & + & + & + & + & + & + & + & + & + & + & - & - & - & - & + & + & - & + \\
\hline Mannitol & + & + & + & + & + & + & + & + & + & + & + & + & + & + & + & + & + & + & - & - & - & - & + & + & + \\
\hline Sorbitol & + & + & + & + & + & + & + & + & + & + & + & + & + & + & + & + & + & + & - & - & - & - & + & + & - \\
\hline Dulcitol & + & + & + & + & + & + & + & + & + & + & + & + & + & + & + & + & + & + & - & - & - & - & + & + & - \\
\hline
\end{tabular}

DW, sterilized distilled water; NT, no treatment; + , positive to metabolize disaccharides and sugar alcohols; - , negative reaction.

${ }^{\mathrm{a}}$ Biovar. 

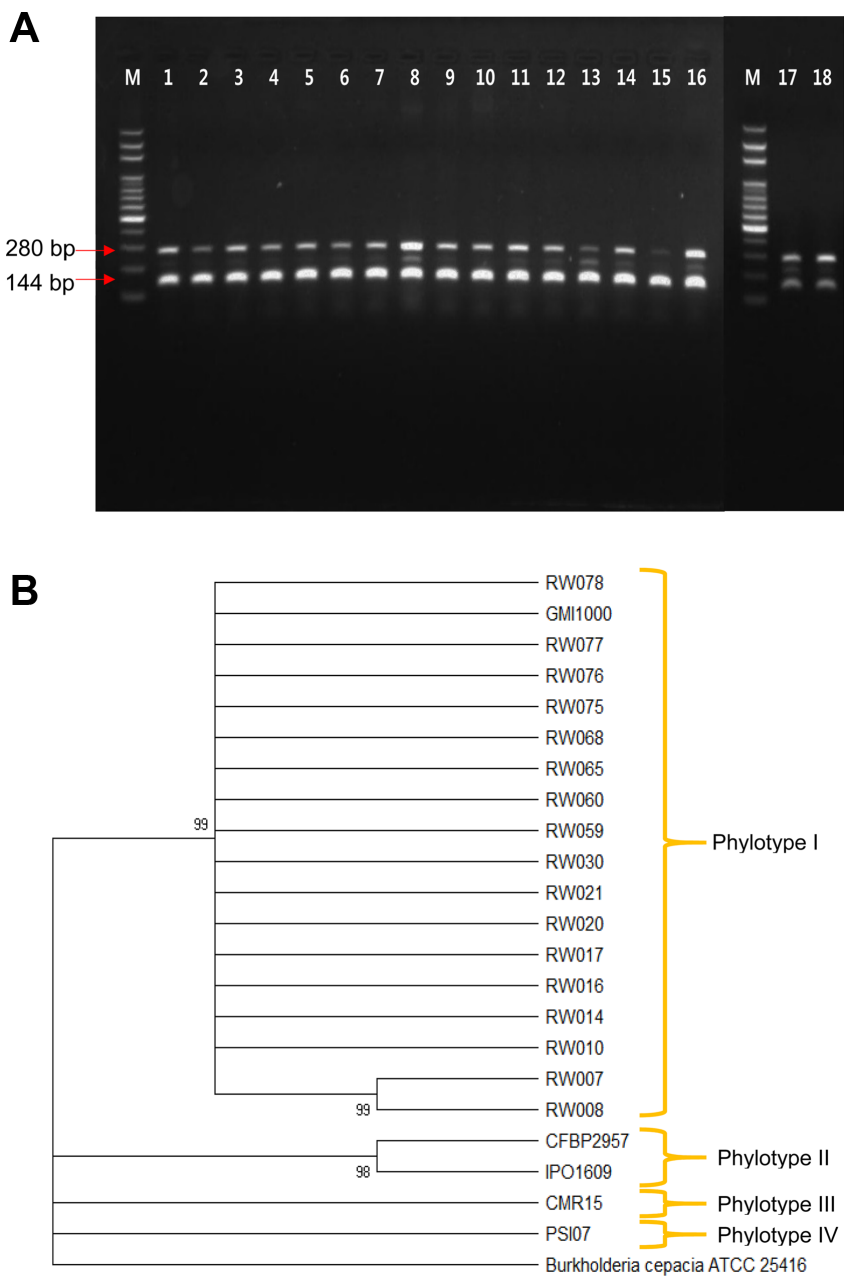

Fig. 2. Phylotype determination of Ralstonia pseudosolanacearum strains isolated from Korean roses. (A) Gel electrophoresis of multiplex PCR products with race and $R$. pseudosolanacearum specific primer sets. Arrows indicate 144 bp bands which are consistent with phylotype I, and 280 bp bands which are consistent with $R$. solanacearum. (B) Phylogenetic tree of $R$. pseudosolanacearum strains isolated from Korean roses and different phylotypes of $R$. solanacearum using partial 16s rRNA gene sequences based on neighbor-joining method. Numbers at each branch indicate bootstrap values greater than 50. Burkholderia cepacia ATCC 25416 was used as the out group.

disaccharides (lactose, maltose, and cellobiose) and three sugar alcohols (mannitol, sorbitol, and dulcitol), whereas KACC 10697 was not able to utilize these three disaccharides. Thus, the 17 strains isolated from Korean roses were determined to be biovar 3 (Table 1).

Phylotype and sequevar determination of $\boldsymbol{R}$. pseudosolanacearum strains isolated from Korean roses. The 17 $R$. pseudosolanacearum strains isolated from Korean roses were amplified as $144 \mathrm{bp}$ amplicons with the phylotype
1 specific primers, but there was no amplification when specific primers for phylotypes 2, 3, and 4 were used. 280 bp amplicon was produced when using the 759/760 primers, which are $R$. solanacearum-specific primers. As such, we are confident there were no false positives in the PCR reactions, and all $17 R$. pseudosolanacearum strains isolated from Korean roses are thought to belong to phylotype I (Fig. 2A). Based on partial sequences of the 16s rRNA gene, all $R$. pseudosolanacearum strains were grouped with GMI1000, which was identified as phylotype I, but segregated from representative strains for phylotypes II, III, and IV (Fig. 2B). Thus, the phylotype determination of the $R$. pseudosolanacearum strains isolated from Korean roses indicated that these strains belong to phylotype I, based on results from PCRs using phylotype specific primers and partial sequences of the 16S rRNA gene.

To analyze the sequevars, a phylogenetic tree was constructed using each partial sequence for egl (accession no. MT380137 of representative strain RW078), fliC (MT380138), and $m u t S$ (MT380139) genes of all $17 R$. pseudosolanacearum strains isolated from Korean roses. All strains clustered with PD7195 and PD7216, and Rs09-161 which were isolated from roses in Netherlands and eggplants (S. melongena) in India (Fig. 3A) (BergsmaVlami et al., 2018; Ramesh et al., 2014), respectively, and as such the sequevar was determined to be I-33. In addition, the tree based on only egl sequences was grouped all 17 R. pseudosolanacearum strains isolated from Korean and Dutch roses, as well as strains CFBP 8468 and CFBP 8469, which caused bacterial wilt on Rodrigues Island into sequevar I-33 (Fig. 3B) (Bergsma-Vlami et al., 2018; Yahiaoui et al., 2017). The trees created based on partial sequences of $e g l, f l i C$, and $m u t S$, and $e g l$ alone were similar to each other; therefore, $17 R$. pseudosolanacearum strains isolated from Korean roses were determined to be sequevar I-33.

Pathogenicity of $\boldsymbol{R}$. pseudosolanacearum strains isolated from Korean roses. Inoculation with the representative strain, RW 078, resulted in the earlier appearance of symptoms in tomato plants than infection with strain KACC 10697. Wilting and blighting of leaves were observed seven days after inoculation, and all inoculated tomato plants eventually died (Fig. 4). In contrast, tomato plants inoculated with strain KACC 10697 showed wilting and browning of the lower leaves until the end of the observation period. However, inoculation with strain KACC 10697 did not produce any visible symptoms on roses of both $\mathrm{cv}$. "Fuego" and "Hera" until 30 days after inoculation, while strain RW 078 produced typical symptoms including wilt- 

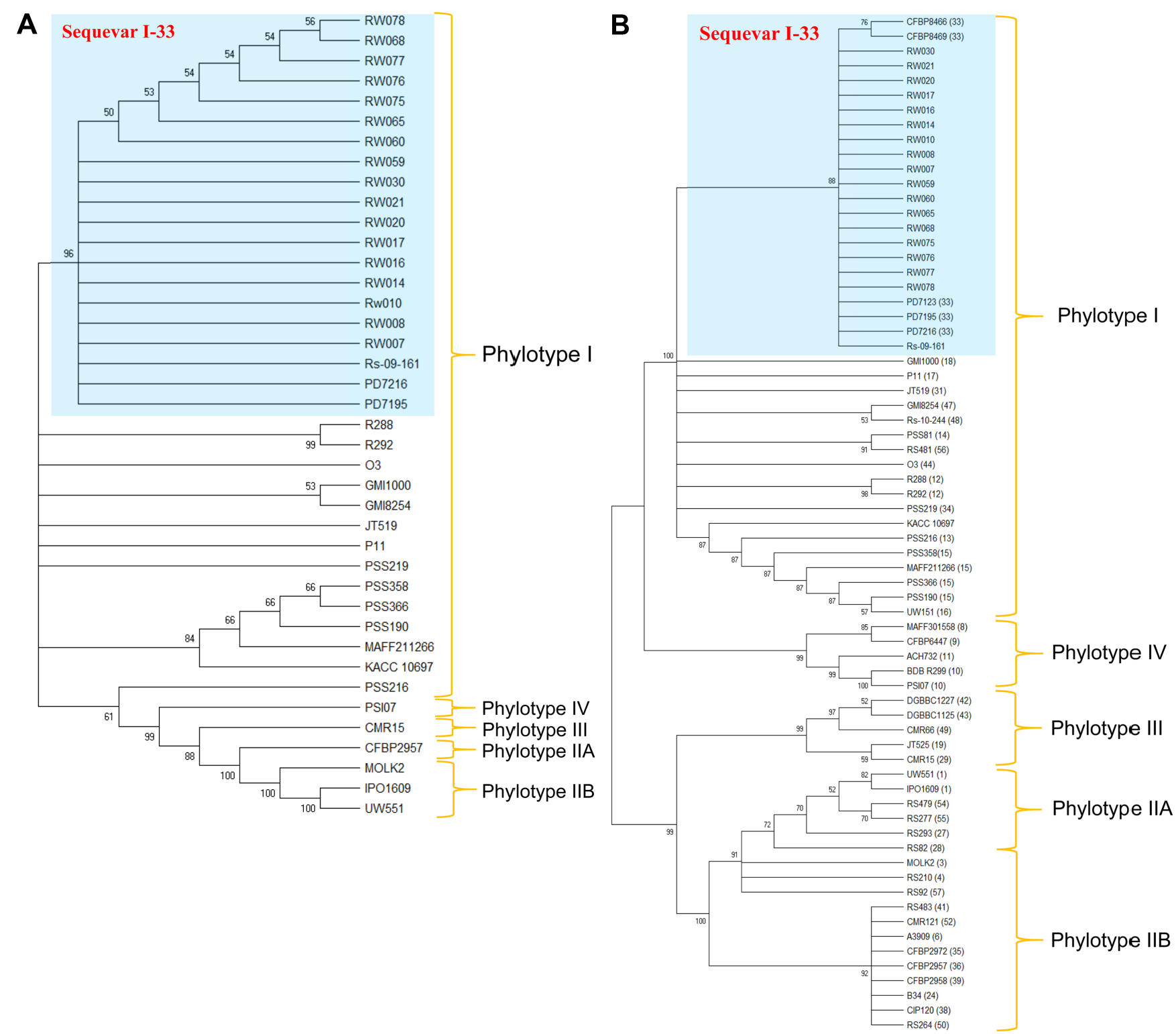

Fig. 3. Sequevars determination of Ralstonia pseudosolanacearum strains isolated from Korean roses and different sequevars of $R$. solanacearum using egl, fliC, and $m u t S$ gene sequences (A) and egl gene sequences only (B) based on neighbor-joining method. Numbers at each branch indicate bootstrap values greater than 50. Parentheses in B indicate the sequevars previous reported.

ing and blighting of leaves from the lower to upper parts of the plants, and symptoms became more pronounced with time (Fig. 5). Interestingly, the leaves of inoculated roses cv. "Fuego" eventually became blighted and died, while inoculated roses of cv. "Hera" did not show any wilting nor blighting symptoms, with the exception of a few yellowing leaves on the lower parts of the plants. Instead, roses of cv. "Hera" had reduced growth compared to the roses inoculated with distilled water (control treatment), indicating that strain RW 078 negatively affected the normal physiological state in these roses. We re-isolated typical colonies from both cv. "Fuego" and "Hera" roses, and confirmed that those isolates were $R$. pseudosolanacearum $(R$. solanacearum phylotype I) strain by multiplex PCR with $R$. solanacearum and phylotype specific primer sets (Supplementary Fig. 1). Therefore, pathogenicity tests conducted on roses inoculated with strain RW 078 satisfied Koch's postulates.

\section{Discussion}

Although R. pseudosolanacearum evaluated in this study 
A

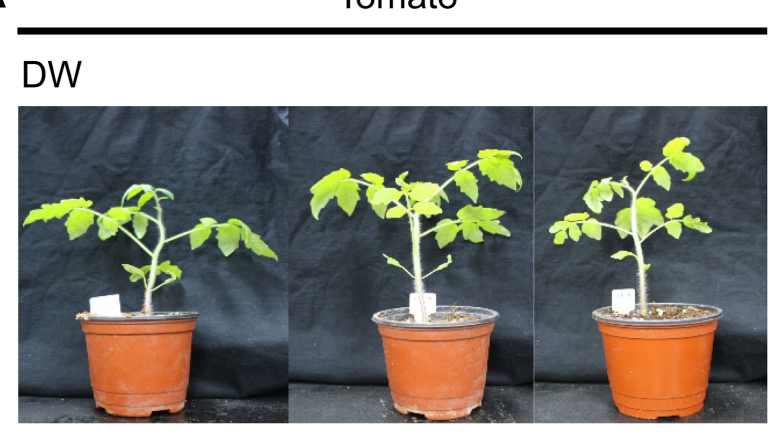

KACC10697

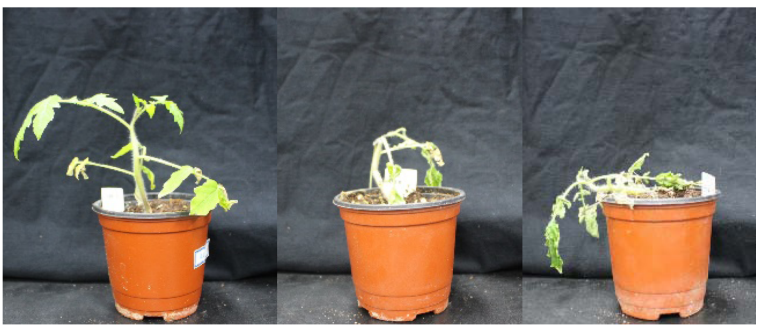

RW078

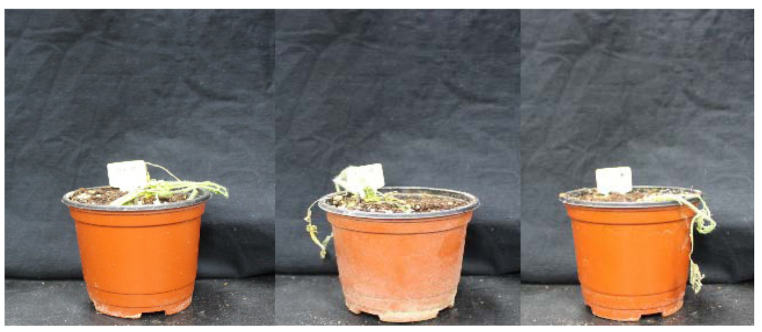

B

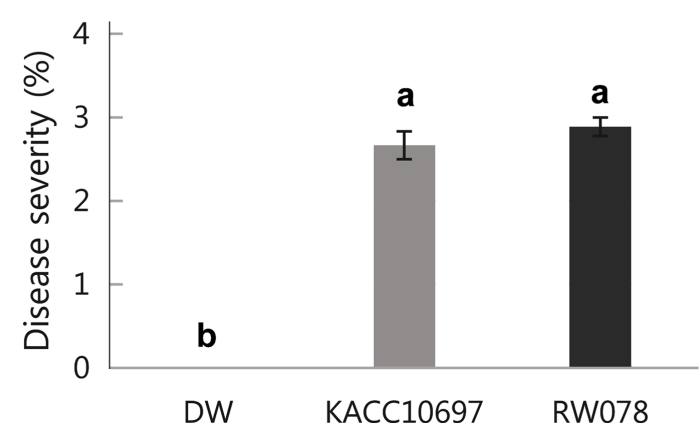

Fig. 4. Pathogenicity of the representative strain, RW 078, Ralstonia pseudosolanacearum isolated from Korean roses, and KACC 10697 on tomato plants cv. "Moneymaker". Photographs were taken at 14 days post inoculation (A). Disease severities are shown the average wilting symptoms on a scale from 0 to 3 (mean $\pm \mathrm{SE}$ ). $n=9$ plants per treated strain. Different letters on bars with error bars indicate significant differences between strain and negative control (DW) according to Duncan's multiple range test at $P<0.05$ (B).

were isolated from only four areas in Korea, diseased roses are frequently reported from areas where roses are culti- vated at a large scale in Korea, indicating that this disease may be prevalent in Korean rose farms, and that no control strategies against it are currently being implemented. This suggests that this disease could spread to all rose farms in Korea in the near future, as roses are multiplied via vegetative propagation plant materials. To address this problem, early diagnosis and field trials are important. However, the early diagnosis of bacterial wilt pathogens on many horticultural crops mainly tomato plants from fields involves cutting stems, which are placed in clean water and then observed for bacterial streaming (Mansfield et al., 2012). However, the accuracy of this method depends on the freshness of the diseased plants. In this study, however, the confirmation of $R$. pseudosolanacearum by bio-PCR analysis of bacterial colonies using primers 759/760 and Nmult21:1F/Nmult22:RR (Fegan and Prior, 2005; Opina et al., 1997) was a suitable, easy, and quick way to identify this pathogenic bacterium.

$R$. solanacearum have the ability to induce disease in more than 450 plant species in 55 families (Hayward and Hartman, 1994), indicating that this species is of economic importance, due to yield loss and deterioration of marketability of various plants (Mansfield et al., 2012). This pathogen is also able to adapt to new hosts and areas, which results in the evolution of its virulence (Remenant et al., 2010). As such, $R$. solanacearum was thought to be a species complex decades ago (Gillings and Fahy, 1994), and more recently this pathogen is referred to as the $R$. solanacearum species complex (RSSC) (Fegan and Prior, 2005), which means RSSC strains have hyper- heterogeneity. Therefore, RSSC strains must be divided into specific classifications which accurately reflect their characteristics. RSSC strains can be phenotypically categorized into classifications such as races (Buddenhagen et al., 1962) and biovars (Hayward, 1964) that reflect pathogenicity in different host plants, and the utilization of three disaccharides and three hexose alcohols, respectively. More recently, RSSC strains have been divided into four phylotypes based on geographical origin using newly developed molecular tools (Fegan and Prior, 2005). RSSC strains have also been divided into three species, namely $R$. solanacearum, $R$. pseudosolanacearum, and $R$. syzygii, in a polyphasic taxonomic study (Safni et al., 2014) as well as proposed sequevars (sequence variant) based on endoglucanase $(e g l)$ gene sequences (Fegan and Prior, 2005).

The causal agent of typical bacterial wilt symptoms on ornamental roses was previously confirmed as $R$. solanacearum (race 1 and biovar 3 ) by Tjou-Tam-Sin et al. (2017a), and Bergsma-Vlami et al. (2018) reported the phylogenetic assignment of $R$. pseudosolanacearum isolat- 
A
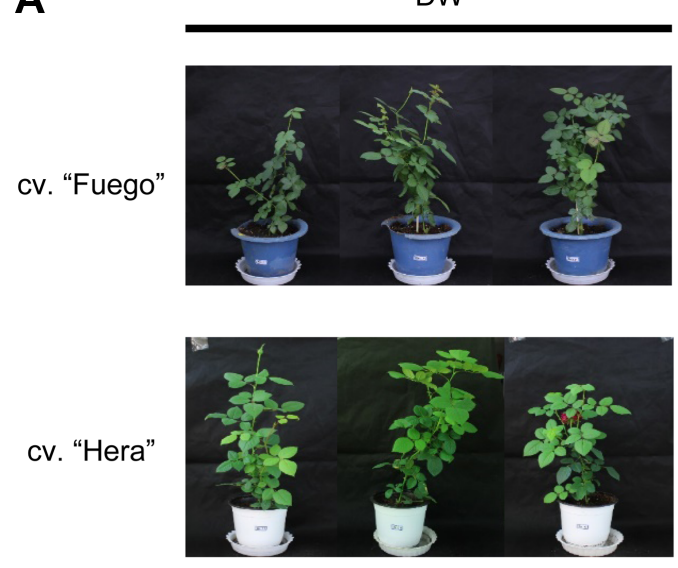

KACC 10697
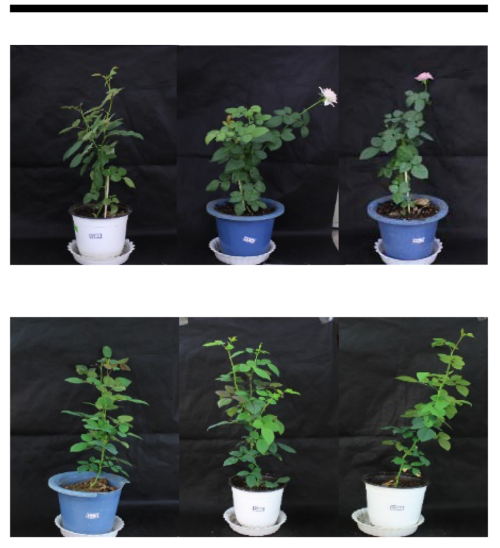

RW078
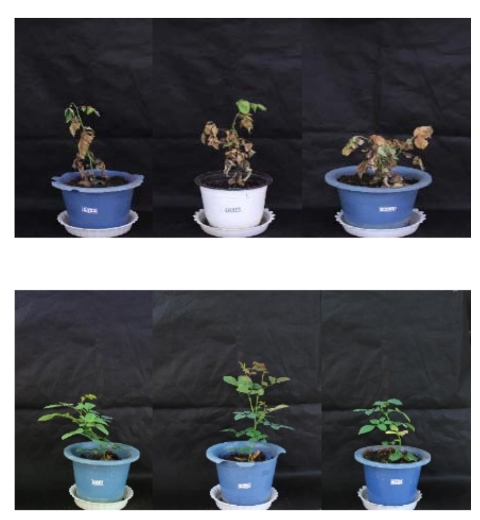

B

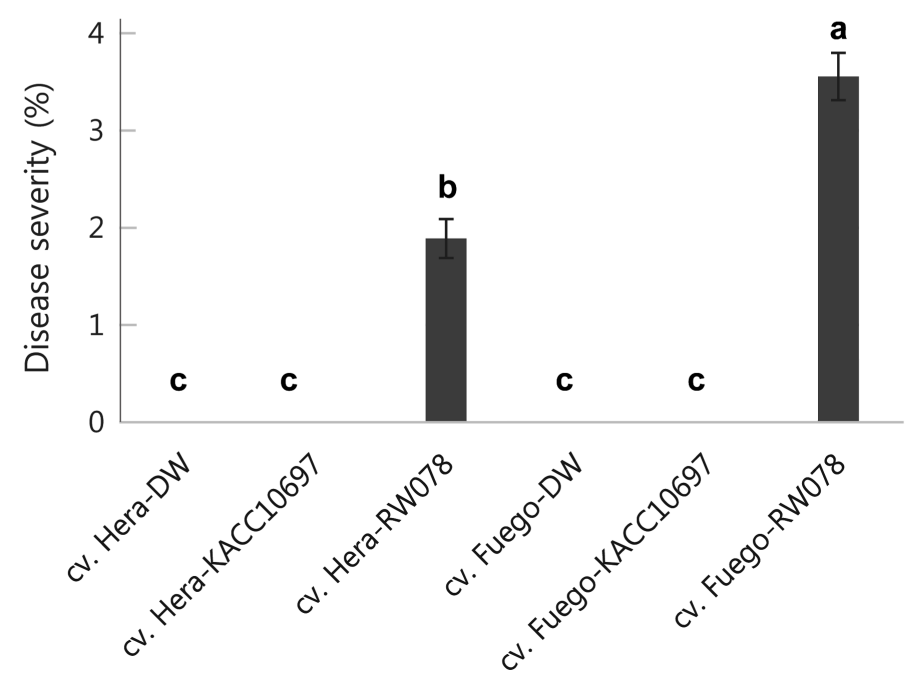

Fig. 5. Pathogenicity of the representative strain, RW 078, Ralstonia pseudosolanacearum isolated from Korean roses, and KACC 10697 on roses cv. "Fuego" and cv. "Hera". Photographs were taken at 30 days post inoculation (A). Disease severities are shown the average wilting symptoms on a scale from 0 to 4 (mean $\pm \mathrm{SE}$ ). $n=9$ plants per treated strain. Different letters on bars with error bars indicate significant differences between strain and negative control (DW) according to Duncan's multiple range test at $P<0.05$ (B).

ed from roses as phylotype I and sequevar 33. In our study, all 17 R. pseudosolanacearum strains isolated from Korean roses were determined to belong to race 1 and biovar 3 by race-specific primers, and the ability to metabolize three disaccharides and three sugar alcohols, respectively. These results suggest the strains evaluated in our study are similar to the $R$. pseudosolanacearum found in roses from the Netherlands. In addition, the phylotype was identified as phylotype I using phylotype specific primers. To clarify the phylotype, we generated a phylogenetic tree based on partial 16s rRNA gene sequences, and all 17 strains isolated from Korean roses were clustered with GMI 1000, which corresponded to phylotype I (Poussier et al., 2000; Safni et al., 2014), indicating that these strains belong to phylotype I. The sequevar determination based on analysis of the $e g l$,
mutS, and fliC genes revealed that all $17 R$. pseudosolanacearum strains isolated from Korean roses clustered with two isolates from roses from the Netherlands and isolate Rs-09-161, which was isolated from eggplants in India in 2009, in agreement with previous reports (Bergsma-Vlami et al., 2018). Furthermore, based on egl gene sequences alone, all $17 R$. pseudosolanacearum strains isolated from Korean roses were clustered with three representative $R$. pseudosolanacearum isolates from roses in the Netherlands, as well as isolated CFBP 8468 and CFBP 8469. The 17 R. pseudosolanacearum isolates collected from Korean roses showing bacterial wilt symptoms belonged to race 1, biovar 3, phylotype I, and sequevar I-33, which exactly matched those of $R$. pseudosolanacearum reported from roses in the Netherlands. These results imply that there is 
little genetic diversity between Korean and Dutch of $R$. pseudosolanacearum, suggesting the recent introduction of this pathogen to Korea via propagation materials from other countries (possibly the Netherlands). On the other hand, it is also possible that there is no progression in evolution of the pathogen, such as in virulence genes and infection capability, which allows the pathogen to survive in new sites

Bergsma-Vlami et al. (2018) also indicated that infected planting materials represent crucial pathways for long distance dispersal of $R$. pseudosolanacearum and Korea imports shoot and root stock of roses from European countries. As such, regulations such as quarantine at the border must be enforced before pathogens can enter the country through imported materials.

Regarding pathogenicity in tomato plants, the representative strain, RW 078, resulted in more severe and earlier symptoms than strain KACC 10697, which was isolated from potatoes in Korea. Additionally, strain RW 078 produced typical symptoms in roses of cv. "Fuego", including wilting and blighting, but inoculation of these roses with strain KACC 10697 resulted no symptoms. There are many reasons why different strains of $R$. solanacearum have different pathogenicities, but we focused on type III secretion system (T3SS) effector repertoires associated with virulence of these two strains. Therefore, we completed whole genome sequences of strain RW 078, compared with GMI1000 and Rs-09-161, which are deposited into phylotype I, and then analyzed T3SS effector repertoires with those strains in which 65 effectors are commonly present among these three strains, while RipAS, RipAT, RipAU, and RipAW are missed or RipBA and RS_T3E_Hyp6 are only added in strain RW 078 (unpublished data). We hope that in the future we will be able to provide a clear picture of T3SS effector repertoires to support rose breeding strategies. Another issue is that $R$. pseudosolanacearum causes different symptoms in different rose cultivars. In our study, roses of cv. "Fuego" were more sensitive to $R$. pseudosolanacearum than roses of cv. "Hera". Thus, resistance and sensitivity tests must be performed because resistant cultivars (such as cv. "Hera" used in this study) could be harboring the pathogen without showing symptoms, which may result in these materials becoming key agents for dispersal of this pathogen.

\section{Acknowledgments}

This research was supported by Rural Development Administration (PJ011871022020), Republic of Korea.

\section{Electronic Supplementary Material}

Supplementary materials are available at The Plant Pathology Journal website (http://www.ppjonline.org/).

\section{References}

Bergsma-Vlami, M., van de Bilt, J. L. J., Tjou-Tam-Sin, N. N. A., Westenberg, M., Meekes, E. T. M., Teunissen, H. A. S. and Van Vaerenbergh, J. 2018. Phylogenetic assignment of Ralstonia pseudosolanacearum (Ralstonia solanacearum Phylotype I) isolated from Rosa spp. Plant Dis. 102:2258-2267.

Buddenhagen, I. W., Sequeira, L. and Kelman, A. 1962. Designations of races in Pseudomonas solanacearum. Phytopathology 52:726.

Elphinstone, J. G., Hennessy, J., Wilson, J. K. and Stead, D. E. 1996. Sensitivity of different methods for the detection of Ralstonia solanacearum in potato tuber extracts. EPPO Bull. 26:663-678.

Fegan, M. and Prior, P. 2005. How complex is the "Ralstonia solanacearum species complex?" In: Bacterial wilt disease and the Ralstonia solanacearum species complex, eds. by $\mathrm{C}$. Allen, P. Prior and A. C. Hayward, pp. 449-461. American Phytopathological Society, St. Paul, MN, USA.

Fegan, M., Taghavi, M., Sly, L. I. and Hayward, A. C. 1998. Phylogeny, diversity and molecular diagnostics of Ralstonia solanacearum. In: Bacterial wilt disease: molecular and ecological aspects, eds. by P. Prior, C. Allen and J. Elphinstone, pp. 19-33. Springer, Berlin, Germany.

Gillings, M. R. and Fahy, P. 1994. Genomic fingerprinting: towards a unified view of the Pseudomonas solanacearum species complex. In: Bacterial wilt: the disease and its causative agent, Pseudomonas solanacearum, eds. by A. C. Hayward and G. L. Hartman, pp. 95-112. CAB International, Wallingford, UK.

Hayward, A. C. 1964. Characteristics of Pseudomonas solanacearum. J. Appl. Bacteriol. 27:265-277.

Hayward, A. C., El-Nashaar, H. M., Nvdegger, U. and De Lindo, L. 1990. Variation in nitrate metabolism in biovars of Pseudomonas solanacearum. J. Appl. Bacteriol. 69:269-280.

Hayward, A. C. and Hartman, G. L. 1994. Bacterial wilt: the disease and its causative agent, Pseudomonas solanacearum. CAB International, Wallingford, UK. 272 pp.

Horita, M., Yano, K. and Tsuchiya, K. 2004. PCR-based specific detection of Ralstonia solanacearum race 4 strains. J. Gen. Plant Pathol. 70:278-283.

Kim, Y. S., Lim, S. R., Kim, J.-W., Lee, H.-J. and Park, D. H. 2019. First report of Ralstonia solanacearum phylotype I causing bacterial wilt on Rosa spp. in Korea. Plant Dis. 103:1407.

Kumar, S., Stecher, G., Li, M., Knyaz, C. and Tamura, K. 2018. MEGA X: molecular evolutionary genetics analysis across computing platforms. Mol. Boil. Evol. 35:1547-1549. 
Lee, Y.-A., Fan, S.-C., Chiu, L.-Y. and Hsia, K.-C. 2001. Isolation of an insertion sequence from Ralstonia solanacearum race 1 and its potential use for strain characterization and detection. Appl. Environ. Microbiol. 67:3943-3950.

Lee, Y.-A. and Khor, C. N. 2003. A novel insertion sequence, ISRso 19, isolated from Ralstonia solanacearum and its application to race differentiation. Plant Pathol. Bull. 12:57-64.

Mansfield, J., Genin, S., Magori, S., Citovsky, V., Sriariyanum, M., Ronald, P., Dow, M., Verdier, V., Beer, S. V., Machado, M. A., Toth, I., Salmond, G. and Foster, G. D. 2012. Top 10 plant pathogenic bacteria in molecular plant pathology. Mol. Plant Pathol. 13:614-629.

Opina, N., Tavner, F., Hollway, G., Wang, J. F., Li, T. H., Maghirang, R., Fagan, M., Hayward, A., Krishnapillai, V., Hong, W., Holloway, B. and Timmis, J. 1997. A novel method for development of species and strain-specific DNA proves and PCR primers for identifying Burkholderia Solanacearum (formerly Pseudomonas Solanacearum). Asia-Pac. J. Mol. Biol. Biotechnol. 5:19-30.

Poussier, S., Prior, P., Luisetti, J., Hayward, C. and Fegan, M. 2000. Partial sequencing of the hrpB and endoglucanase genes confirms and expands the known diversity within the Ralstonia solanacearum species complex. Syst. Appl. Microbiol. 23:479-486.

Ramesh, R., Achari, G. A. and Gaitonde, S. 2014. Genetic diversity of Ralstonia solanacearum infecting solanaceous vegetables from India reveals the existence of unknown or newer sequevars of phylotype I strains. Eur. J. Plant Pathol. 140:543-562.

Remenant, B., Coupat-Goutaland, B., Guidot, A., Cellier, G., Wicker, E., Allen, C., Fegan, M., Pruvost, O., Elbaz, M., Calteau, A., Salvignol, G., Mornico, D., Mangenot, S., Barbe, V., Médigue, C. and Prior, P. 2010. Genomes of three tomato pathogens within the Ralstonia solanacearum species complex reveal significant evolutionary divergence. BMC Genomics 11:379.

Safni, I., Cleenwerck, I., De Vos, P., Fegan, M., Sly, L. and Kappler, U. 2014. Polyphasic taxonomic revision of the Ralstonia solanacearum species complex: proposal to emend the descriptions of Ralstonia solanacearum and Ralstonia syzygii and reclassify current $R$. syzygii strains as Ralstonia syzygii subsp. syzygii subsp. nov., R. solanacearum phylotype IV strains as Ralstonia syzygii subsp. indonesiensis subsp. nov., banana blood disease bacterium strains as Ralstonia syzygii subsp. celebesensis subsp. nov. and $R$. solanacearum phylotype I and III strains as Ralstonia pseudosolanacearum sp. nov. Int. J. Syst. Evol. Microbiol. 64:3087-3103.

Schönfeld, J., Heuer, H., Van Elsas, J. D. and Smalla, K. 2003. Specific and sensitive detection of Ralstonia solanacearum in soil on the basis of PCR amplification of fliC fragment. Appl. Environ. Microbiol. 69:7248-7256.

Thompson, J. D., Gibson, T. J. and Higgins, D. G. 2003. Multiple sequence alignment using ClustalW and ClustalX. Curr. Protoc. Bioinform. 2:2.3.1-2.3.22.

Tjou-Tam-Sin, N. N. A., van de Bilt, J. L. J., Westenberg, M., Bergsma-Vlami, M., Korpershoek, H. J., Vermunt, A. M. W., Meekes, E. T. M., Teunissen, H. A. S. and Van Vaerenbergh, J. 2017a. First report of bacterial wilt caused by Ralstonia solanacearum in ornamental Rosa sp. Plant Dis. 101:378.

Tjou-Tam-Sin, N., van de Bilt, J., Westenberg, M., GorkinkSmits, P., Landman, N. M. and Bergsma-Vlami, M. $2017 \mathrm{~b}$. Assessing the pathogenic ability of Ralstonia pseudosolanacearum (Ralstonia solanacearum phylotype I) from ornamental Rosa spp. plants. Front. Plant Sci. 8:1895.

Wicker, E., Grassart, L., Coranson-Beaudu, R., Mian, D., Guilbaud, C., Fegan, M. and Prior, P. 2007. Ralstonia solanacearum strains from Martinique (French West Indies) exhibiting a new pathogenic potential. Appl. Environ. Microbiol. 73:6790-6801.

Yahiaoui, N., Chéron, J. J., Ravelomanantsoa, S., Hamza, A. A., Petrousse, B., Jeetah, R., Jaufeerally-Fakim, Y., Félicité, J., Fillâtre, J., Hostachy, B., Guérin, F., Cellier, G., Prior, P. and Poussier, S. 2017. Genetic diversity of the Ralstonia solanacearum species complex in the Southwest Indian Ocean islands. Front. Plant Sci. 8:2139. 